\title{
QuESo: a Quality Model for Open Source Software Ecosystems
}

\author{
Oscar Franco-Bedoya ${ }^{1,2}$, David Ameller ${ }^{1}$, Dolors Costal ${ }^{1}$ and Xavier Franch ${ }^{1}$ \\ ${ }^{1}$ Group of Software and Service Engineering, Universitat Politècnica de Catalunya, \\ Campus Nord, Omega building, Jordi Girona Salgado 1-3, Barcelona, Spain \\ ${ }^{2}$ Universidad de Caldas, Manizales, Colombia \\ \{ohernan, dameller, dolors, franch\}@essi.upc.edu \\ http://www.essi.upc.edu/ gessi/
}

Keywords: Quality Model, Software Ecosystem, Quality Measures, Open Source Software

\begin{abstract}
Open source software has witnessed an exponential growth in the last two decades and it is playing an increasingly important role in many companies and organizations leading to the formation of open source software ecosystems. In this paper we present a quality model that will allow the evaluation of those ecosystems in terms of their relevant quality characteristics such as health or activeness. To design this quality model we started by analysing the quality measures found during the execution of a systematic literature review on open source software ecosystems and, then, we classified and reorganized the set of measures in order to build a solid quality model.
\end{abstract}

\section{INTRODUCTION}

Software ecosystems are emerging in the last years as a new way to understand the relationships between software projects, products, and organizations. There are two widespread definitions:

- A software ecosystem is "a set of actors functioning as a unit and interacting with a shared market for software and services. A software ecosystem consists of actors such as independent software vendors (ISV), outsourcers, and customers. A software ecosystem typically is interconnected with institutions such as standardization organizations, open source software communities, research communities, and related ecosystems" (Jansen and Cusumano, 2013).

- A software ecosystem is "a collection of software projects which are developed and evolve together in the same environment" (Lungu et al., 2009).

In the first definition software ecosystems are understood from a holistic business oriented perspective as a network of actors, organizations and companies, while the second definition focuses on technical and social aspects of a set of software projects and their communities. In this paper we try to reconcile both visions and consider the business oriented perspective together with the technical and social perspectives in order to assess software ecosystem quality in its broader sense.
We focus on a particular kind of software ecosystems, i.e., those that are built around an Open Source Software (OSS) initiative (e.g., Android, Gnome, and Eclipse ecosystems), namely OSS ecosystems. We have identified three dimensions of quality in OSS ecosystems: the first dimension is the quality of the software platform in which the projects of the ecosystem are built upon (e.g., the Android ecosystem provides the Android platform used by all the Android mobile apps); the second dimension, as mentioned in Jansen and Cusumano (2013), is the quality of the OSS communities that grow inside the ecosystem and ecosystem's projects (e.g., the Gnome community itself, i.e., the community of the platform, but also the communities of the projects that belong to the ecosystem such as Anjuta, Banshee, and Abi Word communities); the third dimension of quality is inherent to the ecosystems themselves, i.e., the quality derived from the understanding of the OSS ecosystem as a network of interrelated elements (e.g., the number of Eclipse plug-ins and their dependencies between them can be used to assess the ecosystem's interrelatedness).

Assessing the quality of OSS ecosystems is of vital importance because quality assurance is a way to prevent bad decisions, avoid problems, and it allows to verify the compliance with the requirements and the business goals. It can also be used for quality systematic monitoring to provide feedback and execute preventive actions. For example, before deciding 
to integrate a project into an established OSS ecosystem it is crucial to perform a good quality assessment to avoid problems such as inactive user communities, low level of community cohesion, or even synergetic evolution problems, i.e., lack of collaboration between the key developers.

One way to ensure that the quality assessment has covered the most important characteristics of the ecosystem is to use a quality model, "the set of characteristics and the relationships between them which provide the basis for specifying quality requirements and evaluating quality" (ISO/IEC 9126, 2001). Unfortunately, currently there is not any quality model for OSS ecosystems available in the literature, except from some measures distributed among many papers.

To fill this gap, in this paper we present QuESo, a quality model for the quality assessment of OSS ecosystems. To obtain this quality model, first, we searched in the literature for all available measures related to OSS ecosystems, and second, we designed the quality model using both a bottom-up strategy by classifying the measures found, and a top-down strategy by analysing the relationships in the quality characteristics that can be assessed by the measures (e.g., to assess the community activeness we can count the number of changes in the source repository or the number of messages in the mailing lists in a recent period of time).

The rest of the paper is structured as follows: in Section 2 we review the related work; in Section 3 we explain the methods used to construct and design the quality model; in Section 4 we explain the QuESo quality model; in Section 5 we provide examples of real measures and their meaning; in Section 6 we discuss the validity and the observations made in this work; and finally, in Section 7 we provide the conclusions and the future work.

\section{RELATED WORK}

When talking about quality models in the software domain it is inevitable to mention the ISO quality model (ISO/IEC 25000, 2014). This quality model targets the quality of a software product, from three perspectives: internal, external, and quality of use. The specific quality characteristics of the ISO quality model do not cover the important dimensions of OSS ecosystems such as the ones related to the community or the ones related to the health of the ecosystem.

The QualOSS quality model (Soto and Ciolkowski, 2009) gives a good representation for one of the three dimensions covered by QuESo, the OSS community. However we had to extend it with new characteristics that are relevant in the context of OSS ecosystems (see Section 4.2 for details).

As we will explain in Section 3, we have found many papers that, although do not provide a quality model, they propose a good set of measures to evaluate some aspects of OSS ecosystems. We would like to mention some of these works, in particular, the ones that provided the most interesting measures.

- Hartigh et al. (2013) developed a concrete measure tool for evaluating business ecosystems based on the classification made by Iansity and Lieven (2004). They conceptualized the business ecosystem health as financial health and network health based on a set of eight measures.

- Mens and Goeminne (2011) provided a set of measures (e.g., number of commits, total bugs mailing list), by exploring social software engineering, studying the developer community, including the way developers work, cooperate, communicate and share information.

- Neu et al. (2011) presented a web application for ecosystem analysis by means of interactive visualizations. The authors used the application to analysis the GNOME ecosystem study case.

- Kilamo et al. (2012) studied the problem of building open source communities for industrial software that was originally developed as closed source.

Finally we remark the existence of two other secondary studies about software ecosystems (Manikas and Hansen, 2013; Barbosa and Alves, 2011), but in both cases the studies did not have a research question about quality metrics or quality assessment for software ecosystems. Also, it is worth mentioning that as a way to complete our SLR we included the results of these two studies to our SLR (see Section 3.1).

\section{METHOD}

In this section we explain the two methodologies followed in this paper. The first one is related to the way we gathered the measures from the available literature using a Systematic Literature Review (SLR) while the second one is related to the way we designed the QuESo quality model.

\subsection{Systematic literature review}

An SLR is a method to identify, evaluate, and interpret the available research relevant to a particular topic, re- 
search question, or phenomenon of interest (Kitchenham and Charters, 2007).

The protocol described here for the literature review is part of a wider SLR that we are conducting with the goal of identifying the primary studies related to OSS ecosystems. A more detailed explanation of the protocol can be found in Franco-Bedoya et al. (2014).

The research question that addresses the measures and indicators related to the ecosystem quality is: What measures or attributes are defined to assess or evaluate open source software ecosystems?

We defined a search string based on keywords derived from all the SLR research questions: "(OSS OR FOSS OR FLOSS OR Open Source OR Free Software OR Libre Software) AND ecosystem”.

The search strategy used was a combination of sources: searches in digital libraries, manual searches, the inclusion of specific papers from the two secondary studies mentioned in Section 2 and the chapters in a recently published book about software ecosystems (Jansen and van Capelleveen, 2013).

As a result of the SLR, 53 primary studies were selected, from them we identified 17 related to the identification of measures to evaluate the quality of OSS ecosystems. Figure 1 illustrates the SLR selection process.

Once we had collected the measures from the selected papers, we used the following criteria from Hartigh et al. (2013) and Neu et al. (2011) to include them in QuESo:

1. User-friendly and operationalizable: measures should be logical, easy to use and operationalizable into a measurable entity.

2. Non-redundant: when we identified similar measures we selected only one of them, but we kept all the sources for traceability.

After excluding non-operationalizable and merging the similar measures with the previous criteria, we finally selected 68 different measures for the QuESo quality model (note that some of the measures are used to assess more than one characteristic of the quality model).

\subsection{Quality model construction}

There exist several proposals for quality model construction that focus on software quality. Most of them follow top-down strategies (Franch and Carvallo, 2003; Behkamal et al., 2009). In short, they take as a basis a reference quality model such as the ISO quality model (ISO/IEC 25000, 2014), take their quality characteristics as departing point and re-

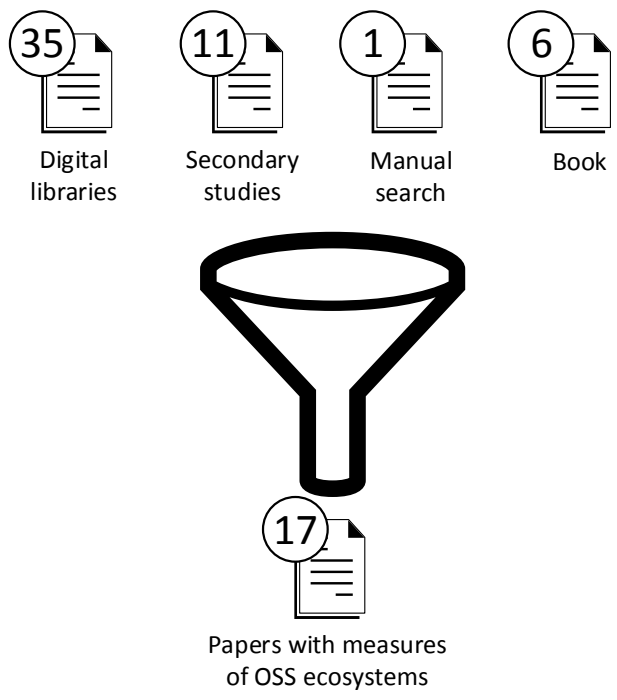

Figure 1: Selection of primary studies.

fine them till they end up with a hierarchy with specific measures at its lower level. Remarkably, the proposal in Radulovic and Garcia-Castro (2011) is mainly bottom-up oriented, i.e., it takes a set of measures as departing point to build the model. For our purposes, a bottom-up approach is the most adequate because: (1) a well-established reference quality model (or even, in its defect, a complete and systematic body of knowledge) for software ecosystems is still missing (Jansen et al., 2013), and (2) there already exist a myriad of specific measures that can be applied to OSS ecosystems and that have been identified in our SLR. Furthermore, although it focuses on the construction of software quality models, we can easily use it to the construction of a quality model for OSS ecosystems.

Radulovic and Garcia-Castro (2011) proposal consists of a clearly defined sequence of steps:

1. To identify basic measures.

2. To identify derived measures.

3. To identify quality measures (by aggregation of basic and derived measures).

4. To identify relationships between measures.

5 . To specify relationships between measures.

6. To define domain-specific quality subcharacteristics.

7. To align quality sub-characteristics with a quality model.

Note that the alignment in the seventh step partly implies top-down reasoning. Quality subcharacteristics that have been previously defined 


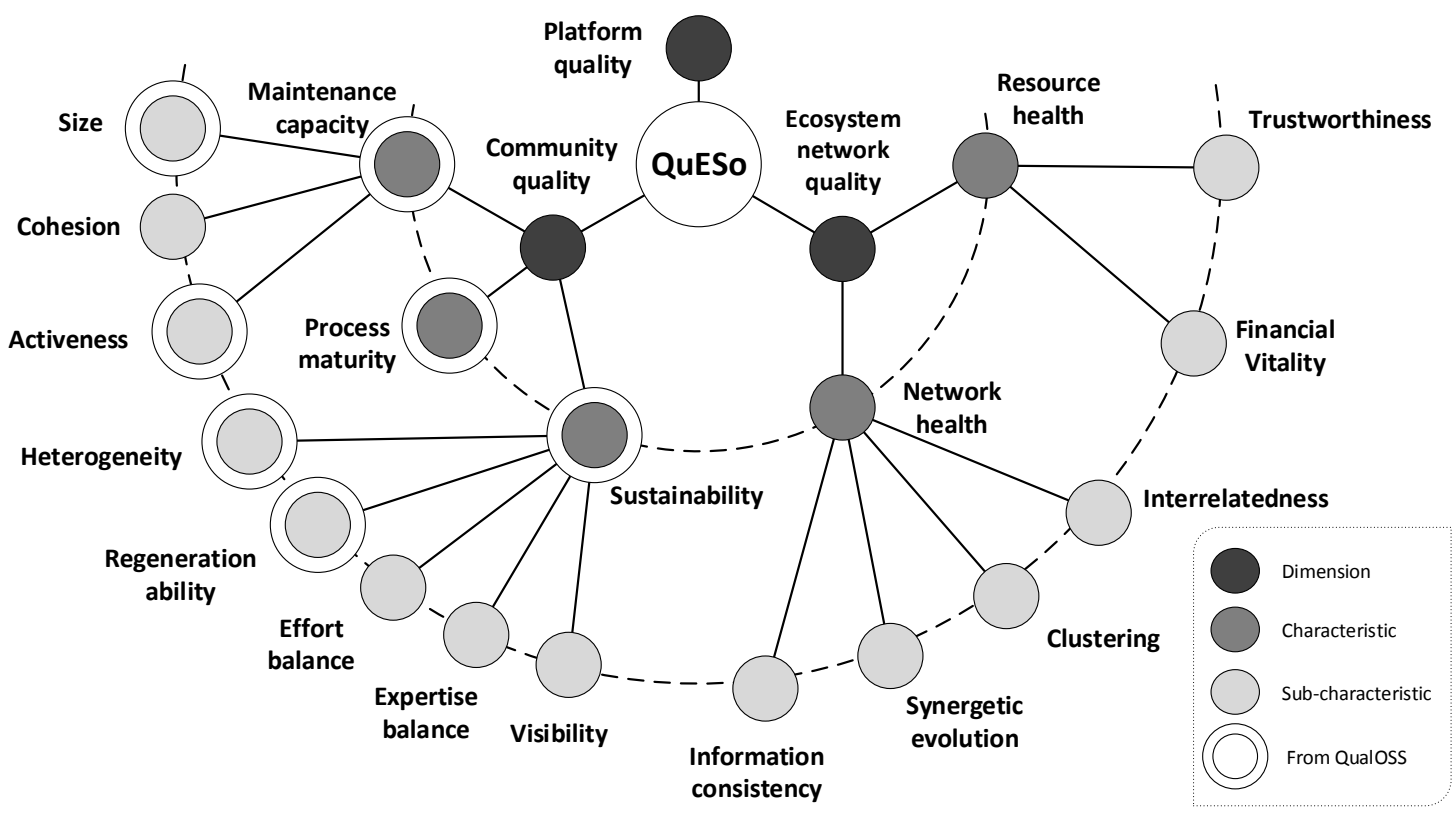

Figure 2: QuESo quality model.

are related to others already specified in the existing model. If needed, some new quality subcharacteristics can be specified, or existing ones can be modified or excluded.

We have followed all the steps of the proposal. In particular, for steps 1 and 2, devoted to identify measures, we have based our work on the SLR described in Section 3.1. The application of step 7 requires the use of a reference quality model. Since, to our knowledge, a quality model for the whole scope of OSS ecosystems is still missing, we have decided to use QualOSS (Soto and Ciolkowski, 2009) which measures the performance of open source communities. Clearly, new quality sub-characteristics emerging from measures related to the ecosystem considered as a whole will have to be specified, since they are not addressed by QualOSS.

\section{QuESo QUALITY MODEL}

In this section we describe the QuESo quality model obtained as a result of the application of the procedure described in Section 3.2. The model is composed of two types of interrelated elements: quality characteristics and measures. Quality characteristics correspond to the attributes of an open source software ecosystem that are considered relevant for evaluation. The quality characteristics are organized in a hierarchy of levels that is described in the rest of this section. For the lack of space, in the tables presented in this paper we have omitted the descriptions. The whole set of measures with their definitions is available in the Appendix. Also, note that we opted to keep the measure names that appear in the primary studies, even that in some cases the name given is not the most appropriate, we discuss about this topic in Section 6.

The quality characteristics in QuESo have been organized in three dimensions: (1) those that relate to the platform around which the ecosystem is built, (2) those that relate to the community (or set of communities) of the ecosystem and (3) those that are related to the ecosystem as a network of interrelated elements, such as projects or companies (see Figure 2).

\subsection{Platform-related quality characteristics}

Platform-related quality characteristics consist of the set of attributes that are relevant for the evaluation of the software platform.

As a result of our SLR, we have observed that the selected papers do not provide measures for evaluating open source platform-related quality characteristics. This fact may indicate that there are not significant differential issues for open source software quality with respect to generic software quality that motivates the need of specific measures.

Then, similarly as done in the QualOSS model, since a mature proposal such as ISO 25000SQuaRE (ISO/IEC 25000, 2014) focuses on generic software quality, QuESo adopts directly the character- 
Table 1: List of measures for maintenance capacity.

\begin{tabular}{ll}
\hline Sub-char. & Measure \\
\hline Size & Number contributors \\
Size & Number of members \\
Size & Number authors \\
Size & Number bug fixer \\
Size & Number of committers \\
Size & Number of core developers \\
Size & Number of nodes and edges \\
Cohesion & Betweenness centrality \\
Cohesion & Cluster of collaborating \\
& developers \\
Cohesion & Ecosystem connectedness \\
Cohesion & Out degree of keystone actors \\
Activeness & Bug tracking activity \\
Activeness & Buildup of assets \\
Activeness & Community effort \\
Activeness & Date of last commit \\
Activeness & Files changed \\
Activeness & Files per version \\
Activeness & Lines added \\
Activeness & Lines changed \\
Activeness & Lines removed \\
Activeness & Mailing list \\
Activeness & Number of commits \\
Activeness & Contributor commit rate \\
Activeness & Developer activity diagrams \\
Activeness & Temporal community effort \\
Activeness & Number of event people \\
\hline
\end{tabular}

istics and sub-characteristics proposed by ISO 25000SQuaRE and this part of the quality model is omitted in the paper.

\subsection{Community-related quality characteristics}

Following the procedure described in Section 3.2, the QuESo proposal for community-related quality characteristics is based both on the set of measures identified in our SLR and on the QualOSS quality model (Soto and Ciolkowski, 2009) (see Figure 2).

QualOSS specifies three community characteristics, namely, maintenance capacity, sustainability and process maturity.

\subsubsection{Maintenance capacity}

Soto et al. define maintenance capacity as the ability of a community to provide the resources necessary for maintaining its products and mention that aspects relevant to it are the number of contributors to a project
Table 2: List of measures for sustainability.

\begin{tabular}{ll}
\hline Sub-char. & Measure \\
\hline Heterogeneity & Geographical distribution \\
Reg. ability & Temporal community effort \\
Reg. ability & New members \\
Effort bal. & Contributor commit rate \\
Effort bal. & Developer activity diagrams \\
Effort bal. & Maximum number of commits of \\
& a developer \\
Effort bal. & Member effort \\
Effort bal. & Member activity rate \\
Effort bal. & Number of activity communities \\
Effort bal. & Number of developer releases \\
Effort bal. & Number of developer projects \\
Effort bal. & Project developer experience \\
Effort bal. & Temporal community effort \\
Effort bal. & Total effort of members \\
Exper. bal. & Expertise view contributor \\
Exper. bal. & Principal member activity \\
Exper. bal. & Relation between categorical \\
& event and developer participation \\
Visibility & Number of event people \\
Visibility & Inquires or feature requests \\
Visibility & Job advertisements \\
Visibility & Number of downloads \\
Visibility & Number of mailing list users \\
Visibility & Number of passive user \\
Visibility & Number of reader \\
Visibility & Number of scientific publications \\
Visibility & Social media hits \\
Visibility & Visibility \\
Visibility & Web page requests \\
\hline &
\end{tabular}

and the amount of time they contribute to the development effort. In order to align maintenance capacity with our identified measures it is refined in three subcharacteristics: size, cohesion and activeness. The size of the community influences its maintenance capacity and can be evaluated by measures such as number of core developers and number of committers. The ability of the community to collaborate defined by its cohesion is also relevant. A measure that can be used to evaluate cohesion is the ecosystem connectedness in the community social network. Finally, the activeness of the community can be evaluated by measures such as bug tracking activity and number of commits.

We have identified 26 measures that can be used to measure the maintenance capacity (see Table 1).

\subsubsection{Sustainability}

Sustainability is the likelihood that a community remains able to maintain the products it develops over 
an extended period of time. According to Soto et al. it is affected by heterogeneity and regeneration ability and, as a result of our measure analysis, we have specified additional sub-characteristics besides them: effort balance, expertise balance and visibility.

The heterogeneity of a community contributes to its sustainability. For instance, if a community is mainly composed of employees of a particular company, there is the risk of the company cutting its financial support. Heterogeneity can be evaluated by measures such as geographical distribution of community members.

Regeneration ability also enhances sustainability since a community that has been able to grow in the past increases its chances of not declining in the future. A measure that we have identified for it is for instance, new members which counts the number of new members of the community at any point of time.

The effort balance is relevant for sustainability i.e., if most of the contribution effort comes from one or a small number of members of the community and it is not uniformly distributed, then its continuity is highly dependent on that small set of members. On the other hand, a balanced effort distribution among all members facilitates its continuity over time. Some measures for effort balance are: number of developer projects and number of developer releases.

In a similar way, the expertise balance among most members of a community is again a way to guarantee its sustainability. A community highly dependent on the expertise of one or a few members suffers from a risky situation. A measure for this is, for instance, expertise view contributor which calculates a contributor expertise based on the number and type of files he changed within a month.

The visibility of a community gives it the capacity of attracting people to contribute and support it if needed. Examples of measures identified for visibility are: number of downloads, social media hits and web page requests.

We have identified 28 measures that can be used to measure the sustainability quality (see Table 2).

\subsubsection{Process maturity}

Process maturity is the ability of a developer community to consistently achieve development-related goals by following established processes. It can be assessed for specific software development tasks with the answers of questions such as: is there a documented process for the task? (Soto and Ciolkowski, 2009). Apparently, this characteristic requires qualitative assessment more than quantitative measures. This is consistent with the results of our SLR since we have not identified measures devoted to evaluate
Table 3: List of measures for resource health.

\begin{tabular}{ll}
\hline Sub-char. & Measure \\
\hline Trustworthiness & Zeta model \\
Trustworthiness & Z-score \\
Financial vitality & Liquidity \\
Financial vitality & Solvency \\
Financial vitality & Network resources \\
\hline
\end{tabular}

process maturity aspects. The absence of measures for process maturity hampers the application of the bottom-up process to further refine this characteristic.

\subsection{Ecosystem network quality characteristics}

Since QualOSS does not address the network-related quality, this part of QuESo is exclusively based on the analysis of measures identified in our SLR.

QuESo proposes two ecosystem network-related characteristics: resource health and network health. In this paper we take as definition for health applied to software ecosystems: longevity and a propensity for growth (Jansen, 2014; Lucassen et al., 2013) .

\subsubsection{Resource health}

Resource health facilitates the combination of value activities from multiple actors to obtain valuecreating end products (Anderson et al., 2009). It is related to the financial health concept defined by Hartigh et al. (2013): "The financial health is a long-term financially based reflection of a partner's strength of management and of its competences to exploit opportunities that arise within the ecosystem and is directly related to the capability of an ecosystem to face and survive disruptions". In the OSS ecosystem case this means that there is a set of partners or actors functioning as a unit and interacting among them. Their relationships are frequently operated through the exchange of information and resources. Two sub-characteristics, particularly relevant to resource health, are the financial vitality and the trustworthiness of the ecosystem.

The financial vitality is the viability and the ability to expand (i.e., robustness, ability to increase size and strength) of the ecosystem (Li et al., 2013). Two examples of measures that evaluate it are liquidity and solvency financial measures. They can be obtained directly, e.g., using balance sheet data of partners, but also indirectly, through the network relations.

Trustworthiness is the ability to establish a trusted partnership of shared responsibility in building an 
Table 4: List of measures for network health.

\begin{tabular}{ll}
\hline Sub-char. & Measure \\
\hline Interrelatedness & Contributor activity graph \\
Interrelatedness & Project activity diagrams \\
Interrelatedness & Networks node connection \\
Interrelatedness & Ecosystem connectedness \\
Interrelatedness & Ecosystem cohesion \\
Interrelatedness & Centrality \\
Interrelatedness & Variety of partners \\
Clustering & Variety in products \\
Clustering & Number community projects \\
Clustering & Number active projects \\
Clustering & Number of files \\
Synergetic evo. & Distribution over the species \\
Synergetic evo. & Ecosystem entropy \\
Synergetic evo. & Ecosystem reciprocity \\
Information & Code vocabulary map \\
consistency & \\
\hline
\end{tabular}

overall open source ecosystem (Agerfalk and Fitzgerald, 2008). Operational financial measures obtained from bankruptcy models (e.g., Z-score and Zeta model) are adequate to measure it because they take short-term and long-term survival into account (Hartigh et al., 2013).

We have identified 5 measures that can be used to measure the resource health quality (see Table 3 ).

\subsubsection{Network health}

Hartigh et al. (2013) define network health as a representation of how well partners are connected in the ecosystem and the impact that each partner has in its local network. Healthy ecosystems show many relations and subsystems of different types of elements that are intensely related (Gamalielsson et al., 2010). Furthermore, in a healthy OSS ecosystem network, these relations are mutualistic (Lundell and Forssten, 2009). Van der Linden et al. (2009) proposed to evaluate the network health of an OSS ecosystem before its adoption. To align network health with the identified measures we have refined it into four sub-characteristics: interrelatedness, clustering, synergetic evolution and information consistency.

Interrelatedness is the ability of the nodes of an OSS ecosystem to establish connections between them. It can be evaluated by measures such as centrality i.e., the number of network relations of a node, and project activity diagrams that allows to obtain the kind of project evolution.

Clustering is the capacity of the species (or nodes) in the entire ecosystem to be classified around its projects. It also enables small OSS projects to come together as a large social network with a critical mass (Scacchi, 2007). Basic measures as number community projects, number of files and variety in products can be used to identify clusters using social network analysis techniques (Lungu et al., 2010).

Synergetic evolution is the ability of the subsystems that constitute the whole ecosystem to form a dynamic and stable space-time structure (Haken, 1980; Li et al., 2013). Measures such as ecosystem entropy and ecosystem reciprocity can be used to evaluate synergetic evolution. The ecosystem entropy measure is based on the definition of software system entropy from Jacobson (2004) who states that it is a measure for the disorder that always increases when a system is modified. Ecosystem reciprocity measures direct and active collaboration between the company and its customers in creating value propositions (e.g., through collaboration with key developers in an OSS community and other companies within the ecosystem) (Glott et al., 2013).

Information consistency is the consistency of the core information elements across the breadth of an ecosystem. The code vocabulary map measure evaluates this sub-characteristic. It consists of a summary of terms used in the source code of the project that can be used to obtain a general overview of the domain language of the project's network.

We have identified 15 measures that can be used to measure the network health quality (see Table 4).

\section{EXAMPLES OF MEASURES}

In this section we provide several examples extracted from the papers selected in the SLR. In particular we have selected the examples that belong to the Gnome software ecosystem. Our intention is to clarify the type of measures that are mentioned in this paper with examples and also to provide some evidence of the feasibility to obtain these measures. As mentioned in Jansen (2014), one of the most habitual problems is the absence of data to calculate the measures.

It is worth mentioning that to perform a complete quality assessment of a software ecosystem we first would need to define the assessment process which is out of the scope of this paper. The quality assessment process will have to deal with, e.g., How are the values of each measure interpreted (i.e., defining what are the good and the bad values)?; How can the measures be merged to provide the assessment for a particular sub-characteristic of the quality model?; or What are the principles to perform the assessment with missing, incorrect, and/or inconsistent measure data? We are will provide the answer to these and 
other questions as part of our future work in this topic.

In the following we present the selected Gnome examples of measure values organized by the characteristics of the QuESo quality model. We omit process maturity because we have not found quantitative measures to evaluate it (see explanation in Section 4.2.3). We also omit resource health measures because examples for them are not reported in the SLR papers for the Gnome ecosystem.

- The maintenance capacity can be evaluated from the number authors measure which gives the amount of people that change files in a project. According to Goeminne and Mens (2013) data, for the Gnome ecosystem there have been 3.500 different people having contributed at least once to at least one of the Gnome projects between 1997 and 2012. The number of commits measure is also relevant. Each commit corresponds to the action of making a set of changes permanent. According to Jergensen and Sarma (2011) approximately 480.000 commits were made in Gnome from 1997 to 2007 .

- A measure for sustainability is the member activity rate which gives a value between 0 and 1 that helps to analyse the effort balance, i.e., a zero value indicates a uniform distribution of the work, which means that each person has the same activity rate while a value of 1 means that a single person carries out all the work. The member activity rate for the Gnome Evince project has had a value between 0,7 and 0,8 from 1999 to 2009 according to Mens and Goeminne (2011).

- The network health of an ecosystem can be evaluated by measures such as number community projects and number active projects. For the Gnome ecosystem, there were more than 1.300 projects between 1997 and 2012 and more than $25 \%$ of them had been active for more than six and a half years. At the lower side of the spectrum, more than $25 \%$ of all projects had been active less than one year (Goeminne and Mens, 2013). Another measure for network health is the contributor activity graph. According to Neu et al. (2011) one of the contributors of the Gnome ecosystem has been working in 499 projects and has more than 15.000 changes between 1998 and 2011.

\section{DISCUSSION}

Some observations were made during the design of this quality model. In the following, the most interesting ones are discussed:
- Completeness: since we followed a mainly bottom-up strategy, the completeness of the quality model depends on how complete the set of measures found in the literature is. In this sense, we would like to remark that our quality model may be not complete by one or more of the following reasons: there may be some papers with relevant measures not included in the SLR because they were not present in digital libraries or because our search string did not find them; another reason could be that some important measures are not yet reported in the literature. In this work, our intention was not to invent new measures but to organize the existing ones into a quality model.

- Quantitative vs. qualitative: the measures found in the literature are mostly quantitative, but a quality assessment may also include qualitative evaluations. For example, we commented in Section 4.2.3 the lack of measures for process maturity because in this case the assessment needs to be done with qualitative evaluations of the community. Since we have focused on quantitative measures, there may be other characteristics of the quality model that require or that may be complemented with qualitative evaluations.

- Unbalanced distribution of measures: just by looking into the measure tables, it is easy to observe that the amount of measures for some characteristics is high (e.g., activeness with 17 measures, visibility with 11 measures) while for other is very low (e.g., heterogeneity with 1 measure, information consistency with 1 measure). This unbalanced situation could be an indicator that more research is needed for the characteristics with a low amount of measures.

- Measure names: we have named the measures included in the QuESo quality model with the same names they are referred to in the SLR papers from where they were extracted. The reason for this is to improve traceability. However, some of those measure names might be ambiguous or misleading because it is not evident from them how the measure is evaluated (e.g., project activity diagrams). To improve measure understandability we have listed their definitions in the Appendix.

\section{CONCLUSIONS}

In this paper we have presented QuESo, a quality model for assessing the quality of OSS ecosystems. This quality model has been constructed following a bottom-up strategy that consisted in searching the 
available measures for OSS ecosystems in the literature and then organize them into several quality characteristics. The presented quality model covers three aspects of OSS ecosystems: the platform, the community, and the ecosystem network; which altogether are a good representation of the most important aspects of an OSS ecosystem.

This quality model can be used as a starting point for the quality assessment of an OSS ecosystem, and it is in our plans for the future work to define a complete quality assessment process (as described in Section 5) and to apply it in a real quality assessment. As consequence new measures may be needed for the assessment, but this is the best way to improve, and complete the quality model, and a way to prove its capabilities in quality assessment.

\section{ACKNOWLEDGMENTS}

This work is a result of the RISCOSS project, funded by the EC 7th Framework Programme FP7/20072013 under the agreement number 318249 . We would also like to thank Carme Quer for her assistance.

\section{REFERENCES}

Agerfalk, P. J. and Fitzgerald, B. (2008). Outsourcing to an Unknown Workforce: Exploring Opensourcing as a Global Sourcing Strategy. Mis Quartely, 32(2):385409.

Anderson, J. C., Narus, J. A., and Narayandas, D. (2009). Business Market Management: Understanding, Creating, and Delivering Value (3rd Edition). Prentice Hall.

Barbosa, O. and Alves, C. (2011). A Systematic Mapping Study on Software Ecosystems. In Proceedings of the $3^{\text {rd }}$ IWSECO, pages 15-26.

Behkamal, B., Kahani, M., and Akbari, M. K. (2009). Customizing ISO 9126 quality model for evaluation of B2B applications. Information and Software Technology, 51(3):599-609.

Franch, X. and Carvallo, J. P. (2003). Using quality models in software package selection. IEEE Software, 20:3441.

Franco-Bedoya, O., Ameller, D., Costal, D., and Franch, X. (2014). Protocol for a systematic literature review on open source-software ecosystems. Technical report, Universitat Politcnica de Catalunya. Available online: www.essi.upc.edu/ gessi/papers/ queso-slrprotocol.pdf.

Gamalielsson, J., Lundell, B., and Lings, B. (2010). The Nagios community: An extended quantitative analysis. In Proceedings of the $6^{\text {th }}$ OSS, pages 85-96. Springer.
Glott, R., Haaland, K., and Bannier, S. (2013). D3.1 Draft Business Model Risk Requirements Report. Deliverable of the RISCOSS FP7 project (grant 318249).

Goeminne, M. and Mens, T. (2013). Software Ecosystems: Analyzing and Managing Business Networks in the Software Industry, chapter Analyzing ecosystems for open source software developer communities, pages 247-275. Volume 1 of (Jansen et al., 2013).

Haken, H. (1980). Synergetics. Naturwissenschaften, 67:121-128.

Hartigh, E., Visscher, W., Tol, M., and Salas, A. J. (2013) Software Ecosystems: Analyzing and Managing Business Networks in the Software Industry, chapter Measuring the health of a business ecosystem, pages 221245. Volume 1 of (Jansen et al., 2013).

Iansiti, M. and Levien, R. (2004). Keystones and dominators: framing operating and technology strategy in a business ecosystem. Technical report, Harvard Business School.

ISO/IEC 25000 (2014). Systems and software engineering - Systems and software Quality Requirements and Evaluation (SQuaRE) - Guide to SQuaRE.

ISO/IEC 9126 (2001). Product quality - Part 1: Quality model.

Jacobson, I. (2004). Object-Oriented Software Engineering: A Use Case Driven Approach. Addison Wesley.

Jansen, S. (2014). Measuring the health of open source software ecosystems: Beyond the scope of project health Information and Software Technology, (Available online).

Jansen, S., Brinkkemper, S., and Michael Cusumano (2013). Software Ecosystems: Analyzing and Managing Business Networks in the Software Industry, volume 1. Edward Elgar Publishing.

Jansen, S. and Cusumano, M. (2013). Software Ecosystems: Analyzing and Managing Business Networks in the Software Industry, chapter Defining software ecosystems: a survey of software platforms and business network governance, pages 13-28. Volume 1 of (Jansen et al., 2013).

Jansen, S. and van Capelleveen, G. (2013). Software Ecosystems: Analyzing and Managing Business Networks in the Software Industry, chapter Quality review and approval methods for extensions in software ecosystems, pages 187-217. Volume 1 of (Jansen et al., 2013).

Jergensen, C. and Sarma, A. (2011). The onion patch: migration in open source ecosystems. In Proceedings of the $19^{\text {th }}$ SIGSOFT and $13^{\text {th }}$ ESEC/FSE, pages 70-80. ACM.

Kilamo, T., Hammouda, I., Mikkonen, T., and Aaltonen, T. (2012). From proprietary to open source - Growing an open source ecosystem. Journal of Systems and Software, 85(7):1467-1478.

Kitchenham, B. and Charters, S. (2007). Guidelines for performing Systematic Literature reviews in Software Engineering Version 2.3. Technical report, Keele University EBSE.

Li, X., Jie, X., Li, Q., and Zhang, Q. (2013). Research on the Evaluation of Business Ecosystem Health. In 
Proceedings of the $6^{\text {th }}$ ICMSEM, pages 1009-1020. Springer.

Lucassen, G., Rooij, K., and Jansen, S. (2013). Ecosystem Health of Cloud PaaS Providers. In Proceedings of the $4^{\text {th }}$ ICSOB, pages $183-194$.

Lundell, B. and Forssten, B. (2009). Exploring health within OSS ecosystems. In Proceedings of the $1^{\text {st }} \mathrm{OS}$ COMM, pages $1-5$.

Lungu, M., Lanza, M., Gîrba, T., and Robbes, R. (2010). The Small Project Observatory: Visualizing software ecosystems. Science of Computer Programming, 75(4):264-275.

Lungu, M., Malnati, J., and Lanza, M. (2009). Visualizing gnome with the small project observatory. In Proceedings of the $6^{\text {th }} M S R$, pages 103-106. IEEE.

Manikas, K. and Hansen, K. M. (2013). Software ecosystems A systematic literature review. Journal of Systems and Software, 86(5):1294-1306.

Mens, T. and Goeminne, M. (2011). Analysing the Evolution of Social Aspects of Open Source Software Ecosystems. In Proceedings of the $3^{\text {rd }}$ IWSECO, pages $1-14$.

Neu, S., Lanza, M., Hattori, L., and D'Ambros, M. (2011). Telling stories about GNOME with Complicity. In Proceedings of the $6^{\text {th }}$ VISSOFT, pages 1-8.

Radulovic, F. and Garcia-Castro, R. (2011). Extending Software Quality Models - A Sample In The Domain of Semantic Technologies. In Proceedings of the $23^{\text {rd }}$ SEKE, pages 25-30.

Scacchi, W. (2007). Free/Open Source Software Development: Recent Research Results and Emerging Opportunities. In Proceedings of the $6^{\text {th }}$ ESEC-FSE, pages 459-468.

Soto, M. and Ciolkowski, M. (2009). The QualOSS open source assessment model measuring the performance of open source communities. In Proceedings of the $3^{\text {rd }}$ ESEM, pages 498-501.

van der Linden, F., Lundell, B., and Marttiin, P. (2009). Commodification of Industrial Software: A Case for Open Source. IEEE Software, 26(4):77-83.

\section{SLR REFERENCES}

[R1] Gamalielsson, J., Lundell, B., and Lings, B. (2010). The Nagios community: An extended quantitative analysis. In Proceedings of the $6^{\text {th }}$ OSS, pages 85-96. Springer.

[R2] Goeminne, M. and Mens, T. (2010). A framework for analysing and visualising open source software ecosystems. In Proceedings of IWPSE-EVOL, pages 42-47. ACM.

[R3] Goeminne, M. and Mens, T. (2013). Software Ecosystems: Analyzing and Managing Business Networks in the Software Industry, chapter Analyzing ecosystems for open source software developer communities, pages 247-275. In (Jansen et al., 2013).
[R4] Hartigh, E., Visscher, W., Tol, M., and Salas, A. J. (2013). Software Ecosystems: Analyzing and Managing Business Networks in the Software Industry, chapter Measuring the health of a business ecosystem, pages 221-245. In (Jansen et al., 2013).

[R5] Jansen, S., Souer, J., Luinenburg, L., and Brinkkemper, S. (2012). Shades of gray: Opening up a software producing organization with the open software enterprise model. Journal of Systems and Software, 85(7):1495-1510.

[R6] Jergensen, C. and Sarma, A. (2011). The onion patch: migration in open source ecosystems. In Proceedings of the $19^{\text {th }}$ SIGSOFT and $13^{\text {th }}$ ESEC/FSE, pages 7080. ACM

[R7] Kabbedijk, J. and Jansen, S. (2011). Steering insight: An exploration of the ruby software ecosystem. In Proceedings of the $2^{\text {nd }} I C S O B$, pages 44-55. Springer.

[R8] Kilamo, T., Hammouda, I., Mikkonen, T., and Aaltonen, T. (2012). From proprietary to open source Growing an open source ecosystem. Journal of Systems and Software, 85(7):1467-1478.

[R9] Lungu, M., Lanza, M., Gîrba, T., and Robbes, R (2010). The Small Project Observatory: Visualizing software ecosystems. Science of Computer Programming, 75(4):264-275.

[R10] Lungu, M., Malnati, J., and Lanza, M. (2009). Visualizing gnome with the small project observatory. In Proceedings of the $6^{\text {th }}$ MSR, pages 103-106. IEEE.

[R11] Mens, T. and Goeminne, M. (2011). Analysing the Evolution of Social Aspects of Open Source Software Ecosystems. In Proceedings of the $3^{\text {rd }}$ IWSECO, pages 1-14.

[R12] Neu, S., Lanza, M., Hattori, L., and D'Ambros, M. (2011). Telling stories about GNOME with Complicity. In Proceedings of the $6^{\text {th }}$ VISSOFT, pages 1-8.

[R13] Scacchi, W. and Alspaugh, T. A. (2012). Understanding the role of licenses and evolution in open architecture software ecosystems. Journal of Systems and Software, 85(7):1479-1494.

[R14] Shao, J., Kuk, G., Anand, S., Morley, J. G., Jackson, M. J., and Mitchell, T. (2012). Mapping Collaboration in Open Source Geospatial Ecosystem. Transactions in GIS, 16(4):581-597.

[R15] Ververs, E., van Bommel, R., and Jansen, S. (2011). Influences on developer participation in the Debian software ecosystem. In Proceedings of the MEDES, pages 89-93. ACM.

[R16] Weiss, M. (2011). Economics of collectives. In Proceedings of the $15^{\text {th }}$ SPLC, pages 39:1-39:8. ACM.

[R17] Yu, L., Cawley, J., and Ramaswamy, S. (2012). Entropy-Based Study of Components in Open-Source Software Ecosystems. INFOCOMP Journal of Computer Science, 11(1):22-31. 


\section{APPENDIX: MEASURE DEFINITIONS}

\begin{tabular}{|c|c|c|}
\hline Measure & Definition & Sources \\
\hline $\begin{array}{l}\text { Amount of inquires or feature } \\
\text { requests }\end{array}$ & $\begin{array}{l}\text { Number of inquire or feedbacks received for the OSS community. Con- } \\
\text { tributions could be corrective, adaptive, perfective or preventive. }\end{array}$ & $\mathrm{R} 8$ \\
\hline Betweenness centrality & Reflects the number of shortest paths that pass through a specific node. & $\mathrm{R} 1$ \\
\hline Bug tracking activity & $\begin{array}{l}\text { Number of comments created in project bug tracker and total number } \\
\text { of actions in the bug tracker. These discussions are often technical in } \\
\text { nature and focus on a specific defect or feature. }\end{array}$ & R6, R8, R15 \\
\hline Buildup of assets & $\begin{array}{l}\text { Total factor productivity over time. Can be measured using individual } \\
\text { company data. }\end{array}$ & $\mathrm{R} 4$ \\
\hline Centrality & $\begin{array}{l}\text { Number of relations clique memberships. Number of individual net- } \\
\text { work relations of a partner. The more central partner is the most per- } \\
\text { sistent. When the partners are in clique or cluster, its persistence is } \\
\text { considered high. Because is regarded as a secure environment. }\end{array}$ & $\mathrm{R} 1, \mathrm{R} 4, \mathrm{R} 7$ \\
\hline $\begin{array}{l}\text { Cluster of collaborating } \\
\text { developers }\end{array}$ & $\begin{array}{l}\text { The nodes are developers and the edges between them represent projects } \\
\text { on which they collaborated. They both make modifications to the } \\
\text { project for at least a certain number of times. }\end{array}$ & R9, R10 \\
\hline Code vocabulary map & $\begin{array}{l}\text { Summary of terms used in the source code of the project. The vocab- } \\
\text { ulary map is a tool for the developer who wants to obtain a general } \\
\text { overview of the domain language of a project. }\end{array}$ & R9 \\
\hline Community effort & The combined effort of all members belonging to community. & R3 \\
\hline Contributor activity graph & The contributor distribution at ecosystem level. & $\mathrm{R} 12$ \\
\hline Contributor commit rate & Average between first and last commit. & $\mathrm{R} 12$ \\
\hline Date of last commit & Date of last commit of a project/community. & $\mathrm{R} 11$ \\
\hline Developer activity diagrams & Give an overview of the contributors daily activity within an ecosystem. & $\mathrm{R} 12$ \\
\hline Distribution over the species & $\begin{array}{l}\text { Variety measure for niche creation factor. The equality of the division } \\
\text { of partners over the species. E.g., the distribution between numbers of } \\
\text { resellers, number of system integrators, numbers of OEM's. }\end{array}$ & $\mathrm{R} 4$ \\
\hline Ecosystem cohesion & $\begin{array}{l}\text { Number of relations present in a subgroup/maximum possible of rela- } \\
\text { tion among all the nodes in the sub-group. }\end{array}$ & $\mathrm{R} 4$ \\
\hline Ecosystem connectedness & $\begin{array}{l}\text { Number of relations as a proportion of the theoretically maximum num- } \\
\text { ber of relations in all ecosystem. Is a metric of connectedness. Is a } \\
\text { property that keeps communities structure safe from risks, guaranteeing } \\
\text { their well-being and health. }\end{array}$ & $\mathrm{R} 4$ \\
\hline Ecosystem Entropy & $\begin{array}{l}\text { The second law of thermodynamics, in principle, states that a closed } \\
\text { system's disorder cannot be reduced, it can only remain unchanged or } \\
\text { increase. A measure of this disorder is entropy. This law also seems } \\
\text { plausible for software systems; as a system is modified, its disorder, } \\
\text { or entropy, always increases. This is known as software entropy. Can } \\
\text { be viewed as being similar to the measurement of the existence of or- } \\
\text { der or disorder among the participating software components, software } \\
\text { products, or software organizations. }\end{array}$ & $\mathrm{R} 17$ \\
\hline Expertise view contributor & $\begin{array}{l}\text { Visualization about a contributor expertise based on file extensions } \\
\text { (number and type of files changed within a month). }\end{array}$ & $\mathrm{R} 12$ \\
\hline Files changed & Number of files that has been changed. & $\mathrm{R} 12$ \\
\hline Files per version & Number of files per version. & $\mathrm{R} 11$ \\
\hline Geographical distribution & Geographical distribution of community members. & R9 \\
\hline Job advertisements & Number of job advertisements on the project/community. & $\mathrm{R} 8$ \\
\hline Lines added & Lines added. & $\mathrm{R} 12, \mathrm{R} 7$ \\
\hline Lines changed & Lines changed. & $\mathrm{R} 12$ \\
\hline Lines removed & Lines removed. & $\mathrm{R} 12$ \\
\hline Liquidity & $\begin{array}{l}\text { Is a metric for the robustness factor: survival rates. Provide an indica- } \\
\text { tion whether a partner is able to meet its short-term obligations. Can } \\
\text { be measured with: financial status of a partner; counting the number of } \\
\text { new members in a business ecosystem. }\end{array}$ & $\mathrm{R} 4$ \\
\hline Mailing list & $\begin{array}{l}\text { Number of messages posted to project mailing lists and the number of } \\
\text { responses obtained from those messages. }\end{array}$ & $\begin{array}{l}\text { R1, R11, R15, } \\
\text { R6, R2 }\end{array}$ \\
\hline $\begin{array}{l}\text { Maximum number of commits } \\
\text { of a developer }\end{array}$ & The size and density of a contributor in a project. & $\mathrm{R} 12$ \\
\hline Member activity rate & Activity rate 1 means that a single person carries out all the work. & $\mathrm{R} 11$ \\
\hline
\end{tabular}


Member effort

Network resources

Networks node connection

New members

Number active projects

Number authors

Number bug fixer

Number committers

Number of activity

communities

Number of commits

Number of community project

Number of contributors

Number of core developers

Number of developer releases

Number of developer projects

Number of downloads

Number of event people

Number of files

Number of mailing list users

Number of members

Number of nodes and edges

Number of passive user

Number of reader

Number of scientific

publications

Out degree of keystone actors

Principal member activity

Project activity diagrams

Project developer experience Reciprocity of the ecosystem Relation between categorical event and developer participation

Social media hits

Solvency

Temporal community effort

Total effort of members

Variety in products

Variety of partners

Visibility

Web page requests

Zeta model

Z-score
The effort of member $m$ in community $c$.

Measure for delivery innovations factor of productivity. They can be measured directly, e.g., using balance of partners, but also indirectly, through the network relations.

Connections between central and non-central species or partners.

Counting the number of new members at any point in time.

Number active projects.

Number of authors for projects. Author can change files in a project.

Number bug fixers in the community.

Number of committers per project.

The number of activity communities in which member $m$ is involved.

Total number of commits containing source code, documentation, and translation. Average number of commits per week (project/community). Number of projects built on top of the platform of a community.

Total of contributors per project.

Core developer contribute most of the code and oversee the design and evolution of the project.

Number of releases that a developer has been active on a project.

Number of projects of a developer.

Number of downloads from the official community portal or mirrors.

The number of people participating in project events and meetings gives direct information on the activity in the community.

Files during projects life.

Number of users subscribed to the project mailing list.

The number of activity members involved in community $c$.

Number of nodes and edges.

Passive users in the community.

Number of readers in the community.

Number of scientific publications mentioning the community.

R15, R9, R11,

$\mathrm{R} 12, \mathrm{R} 3, \mathrm{R} 14$

R8, R3

$\mathrm{R} 8, \mathrm{R} 12$

$\mathrm{R} 1$

R6

$\mathrm{R} 12$

$\mathrm{R} 8, \mathrm{R} 7$

$\mathrm{R} 8$

R14, R11

R8

R3, R5, R16

$\mathrm{R} 1$

R8

R8

R8

Is defined for this specific case as someone who has a lot of developers

he works with and also plays a large role in the software ecosystem.

The principal activity of a member $m$ for a given time $t$. Community $c$ for which $m$ carried out the most effort.

Allow identify the project evolution comparing six metrics; calculating the contributors involvement distribution.

Total number of releases in which the developer was active.

(definition not provided).

Relation between categorical event and developer participation.

Number of hits the project gets in the social media and blogs.

R8, R7

Value creation measure for niche creation. Can be measured by standard metrics such as revenue share or profit share of newly introduced products or technologies. An alternative is to look at the build-up of partner equity.

The combined effort of all members belonging to community $c$ during time period $t$.

Total effort done by a particular community member $m$ in a set of communities $C$.

Measure for the variety factor of niche creation. The variety in products offered by the partner depends on alliances with other partners. Euclidean distances towards the overall mean of the business ecosystem can be used to measured most of these variety of scores.

Covariance with market indicates the variety of different partners a partner has.

Tell us something about the centrality of a partner in the market. Popularity of the partner.

Total request to OSS community web page.

Bankruptcy classification score model.

Bankruptcy model to test the creditworthiness and solvency of partners.
R4

$\mathrm{R} 4, \mathrm{R} 13$ 